\title{
Presencia del componente de la zona de transformación del cuello uterino en citologías anormales en la Clínica de Maternidad Rafael Calvo de Cartagena (Colombia) entre 2009 y 2012
}

\author{
Presence of transformation zone component of the cervix in abnormal Pap \\ tests in the Clinica de Maternidad Rafael Calvo de Cartagena (Colombia) \\ from 2009 to 2012
}

\author{
Lía Barrios-García $M D^{1}$, \\ Inés Benedetti-Padrón $\mathrm{MD}^{2}$, Luis Alvis-Estrada $M \mathrm{MS}^{3}$
}

Introducción: se ha determinado que es más probable la detección de lesiones de alto grado del cuello uterino en extendidos citológicos con presencia de componente de la zona de transformación; no obstante, la relación entre las tasas de muestreo de esta zona y la detección de alteraciones citológicas es controversial. Objetivo: determinar la presencia del componente de la zona de transformación en citologías cervicales convencionales anormales. Materiales y métodos: se realizó un estudio de corte transversal de todos los extendidos cervicovaginales anormales de la Unidad de Patología Cervical de la E.S.E. Clínica de Maternidad Rafael Calvo de Cartagena (Colombia) entre 2009 y 2012. Se realizó una segunda lectura de la presencia de componente de la zona de transformación y su coexistencia con células anormales en el mismo campo microscópico, a diferentes

\footnotetext{
${ }^{1}$ Médica, especialista en Patología y especialista en Docencia universitaria. Profesora titular, Facultad de Medicina, Universidad de Cartagena. Grupo de Investigación Histopatología, Cartagena, Colombia. Correspondencia: Carrera 50C N. ${ }^{\circ}$ 24-120. Facultad de Medicina, Universidad de Cartagena, Campus de la salud Zaragocilla. Teléfono: 5756698176 ext. 129. Correo electrónico: Ibarriosg@unicartagena.edu.co 2 Médica, especialista en Patología. Profesora titular, Facultad de Medicina, Universidad de Cartagena. Grupo de Investigación Histopatología, Cartagena, Colombia.

${ }^{3}$ Economista, MSc en Salud Pública. Profesor asistente, Facultad de Enfermería, Universidad de Cartagena. Grupo de Investigación Histopatología, Cartagena, Colombia.

Conflicto de intereses: los autores declaran que no tienen conflicto de intereses Medicina \& Laboratorio 2016; 22: 355-364

Módulo 19 (Investigación), número 46. Editora Médica Colombiana S.A. $2016^{\circ}$

Recibido el 15 de julio de 2016; aceptado el 28 de agosto de 2016
} 
aumentos, comparando la concurrencia de ambos tipos celulares en lesiones intraepiteliales escamosas de alto y bajo grado. Resultados: se incluyeron 1.287 citologías anormales, $74,7 \%$ con componente de la zona de transformación. A mayor grado de lesión se encontró mayor porcentaje de extendidos con dicho componente. La coexistencia de células endocervicales y anormales en el mismo campo microscópico fue mayor en las lesiones de alto grado en comparación con las de bajo grado $\left(p=0,000, X^{2}\right)$. Conclusiones: hay mayor frecuencia de extendidos citológicos anormales con presencia de células de la zona de trasformación del cérvix. Su presencia es más frecuente en los extendidos con lesiones de alto grado, donde las células endocervicales o metaplásicas se encuentran íntimamente relacionadas con las anormales.

Palabras clave: prueba de Papanicolaou, neoplasia intraepitelial del cuello uterino, técnicas citológicas.

Introduction: It has determined that the detection of high grade squamous intraepithelial lesion is more probably in cytological smears with presence of transformation zone component, but the relation between sampling rates of this zone and detection of cytological abnormalities is controversial. Objective: To determine the presence of transformation zone component in abnormal cervical smears. Material and methods: It was made a cross-sectional study of all abnormal cervical-vaginal smears studied in Cervical Pathology Unit of ESE Clinica de Maternidad Rafael Calvo de Cartagena (Colombia) between 2009 and 2012. A second reading of the presence of transformation zone component it was made, as well as of their coexistence with abnormal cells in the same microscopic field at different magnifications, comparing the concurrence of both cell types in low and high-grade squamous intraepithelial lesions. Results: 1,287 abnormal cervical smears were included, $74.7 \%$ of them with transformation zone component. To higher degree of lesion, higher percentage of smears with this component was found. Coexistence of endocervical and abnormal cells in the same microscopic field was higher in the high-grade squamous intraepithelial lesions compared with those with low grade $\left(p=0.000 X^{2}\right)$. Conclusions: There is a higher frequency of abnormal cervical smears with presence of transformation zone component of the cervix. Their presence is more frequent in the smears with high-grade lesions, where endocervical cells or metaplastic are closely related to abnormal cells.

Key words: Papanicolaou test, cervical intraepithelial neoplasia, cytological techniques.

Barrios-García L, Benedetti-Padrón I, Alvis-Estrada L. Presencia del componente de la zona de transformación del cuello uterino en citologías anormales en la Clínica de Maternidad Rafael Calvo de Cartagena (Colombia) entre 2009 y 2012. Medicina \& Laboratorio 2016; 22: 355-364. 
$\mathrm{E}$ I cáncer de cuello uterino continúa siendo una de las principales causas de mortalidad por cáncer y ocupa el segundo lugar en incidencia de cáncer en mujeres en Colombia [1]. A pesar de ser un tumor prevenible y de la implementación del programa de detección temprana mediante citología exfoliativa, no se ha mostrado una tendencia al descenso de tales cifras, en contraste con la observada en los países industrializados [2].

El objeto de la citología exfoliativa es obtener células, principalmente de la unión escamocolumnar; área en que se desarrolla con mayor frecuencia el cáncer [3-6]. La citología tiene como limitación que la sensibilidad varía entre $51 \%$ y 55,2\% y cuando se enfoca en lesiones de alto grado entre un $45,5 \%$ y un $64,7 \%$ [7-9]. Una citología tomada adecuadamente debe incluir células escamosas y células endocervicales o metaplásicas, indicadoras de muestreo de la zona de transformación del cérvix, donde se considera que se originan la mayoría de las alteraciones epiteliales $[10,11]$ y cuya presencia aumenta la sensibilidad del método [12-14].

Teniendo en cuenta lo anterior, el Sistema Bethesda, establecido para estandarizar los reportes de citología cervical, desde su inicio en 1988 ha considerado la información sobre la presencia o ausencia del componente de la zona de transformación como un elemento obligatorio en el reporte del resultado, incluso incluyó la presencia de dicho componente como requisito para determinar una muestra como "satisfactoria" [15].

En la revisión de 1991 del Sistema Bethesda se incluyó la categoría "satisfactoria, limitada por" para muestras que cumplen con todos los requisitos, excepto la presencia de células endocervicales o metaplásicas [16]. No obstante, esta terminología generó confusiones en la práctica clínica, por lo que en el 2001 Bethesda mantuvo la categoría "satisfactoria para evaluación", pero eliminó la opción "satisfactoria, limitada por", considerando satisfactorios los extendidos sin representación de la zona de transformación, pero, a la vez, resaltando la importancia de obtener muestra de la misma y de incluir en el informe su presencia o ausencia [17].

La importancia de la representación de la zona de transformación del cuello uterino en los extendidos citológicos sigue vigente; aunque es aceptado que hasta un $20 \%$ de los extendidos citológicos de mujeres entre los 20 y 50 años no tengan dicha representación [18]. Se ha informado que es más probable la detección de lesiones de alto grado, que son las que tienen un riesgo real de progresar a cáncer [19], en extendidos citológicos con presencia de componente de la zona de transformación que sin este; no obstante, los resultados son controversiales respecto a una relación significativa entre las tasas globales de muestreo de esta zona y las tasas de detección de alteraciones citológicas [10,20-26].

El objetivo de este estudio fue determinar la presencia de células endocervicales o metaplásicas del cérvix en extendidos citológicos anormales. Además, se pre- 
tendió encontrar evidencia a favor de la hipótesis de que a mayor grado de lesión aumenta el porcentaje de presencia de dicho componente y que hay mayor cercanía entre las células anormales y endocervicales en los extendidos con lesión de alto grado que en aquellos con lesión menor.

\section{Materiales y métodos}

\section{Tipo y población de estudio}

Se realizó un estudio de corte transversal en el que se incluyeron los extendidos de citología cervical estudiados en la Unidad de Patología Cervical de la E.S.E. Clínica de Maternidad Rafael Calvo de Cartagena; entidad de nivel II de complejidad que atiende a la población de bajo nivel socioeconómico, afiliada al régimen subsidiado público en la ciudad de Cartagena de Indias, Colombia.

Se tomaron todos los extendidos cervicovaginales anormales, informados según el Sistema Bethesda (2001) como: atipias en células escamosas de significado indeterminado (ASC-US), atipias en células escamosas que podrían indicar lesión de alto grado (ASC-H), atipias en células glandulares (AGC), lesión intraepitelial escamosa de bajo grado (LIEBG) y lesión intraepitelial escamosa de alto grado (LIEAG) [17], realizados entre enero de 2009 y diciembre de 2012, con datos documentados en la base de datos del Laboratorio de Patología de la E.S.E. Clínica de Maternidad Rafael Calvo de Cartagena.

Se excluyeron los extendidos anormales con antecedentes o diagnóstico de cáncer y los provenientes de pacientes histerectomizadas. La información fue obtenida en forma anónima, sin tener en cuenta los datos personales y el estudio fue aprobado por el Comité ético institucional.

Todos los extendidos fueron revisados e informados bajo el Sistema Bethesda (2001), por los patólogos institucionales y se registraron en la base de datos como satisfactorios (negativos o anormales) e insatisfactorios.

\section{Evaluación de la presencia}

\section{de componente de la zona de transformación}

Se realizó una segunda lectura de todos los extendidos anormales incluidos en el estudio para evaluar la presencia de componente de la zona de transformación. La lectura, realizada por una citotecnóloga y dos patólogos investigadores, se llevó a cabo en un microscopio óptico de luz, binocular, marca Nikon ${ }^{\circledR}$ (Nikon Corporation, Tokio, Japón), bajo los objetivos de $4 \mathrm{X}, 10 \mathrm{X}$ y $40 \mathrm{X}$.

Se consideró positiva la representación de la zona de transformación cuando se identificaron mínimo 10 células endocervicales o metaplásicas. Cada extendido fue evaluado anotando la categoría citológica [17], la presencia o ausencia de 
material endocervical, la coexistencia de células endocervicales y anormales en el mismo campo microscópico a diferentes aumentos $(4 \mathrm{X}, 10 \mathrm{X}$ y $40 \mathrm{X})$ y la presencia de atrofia. Tras esta relectura fueron eliminados los extendidos atróficos por la dificultad de identificar las células endocervicales en los mismos [27].

\section{Determinación de la cercanía entre las células endocervicales y las anormales}

Para la determinación de la asociación del grado de cercanía entre las células endocervicales y las anormales en las lesiones de alto grado se conformaron dos grupos: a) los extendidos anormales con células endocervicales con resultado de atipias en células escamosas de significado indeterminado y lesión de bajo grado y b) los extendidos anormales con células endocervicales con atipias en células escamosas que podrían indicar lesión de alto grado y con lesión de alto grado. En cada uno de ellos se evaluó la presencia de ambos tipos de células, endocervicales y anormales, en el mismo campo microscópico, usando objetivos de diferente poder (4X, 10X y 40X).

Es importante tener en cuenta que a medida que aumenta el poder del objetivo disminuye el tamaño del campo microscópico y, de esta manera, la concurrencia de ambos tipos celulares en un campo microscópico con objetivo de alto poder (40X) corresponde al mayor grado de cercanía entre ellas (véase figura 1 ).

Posteriormente, se comparó la concurrencia de ambos tipos celulares en los diferentes poderes de aumento microscópico entre los dos grupos de extendidos mencionados, debido

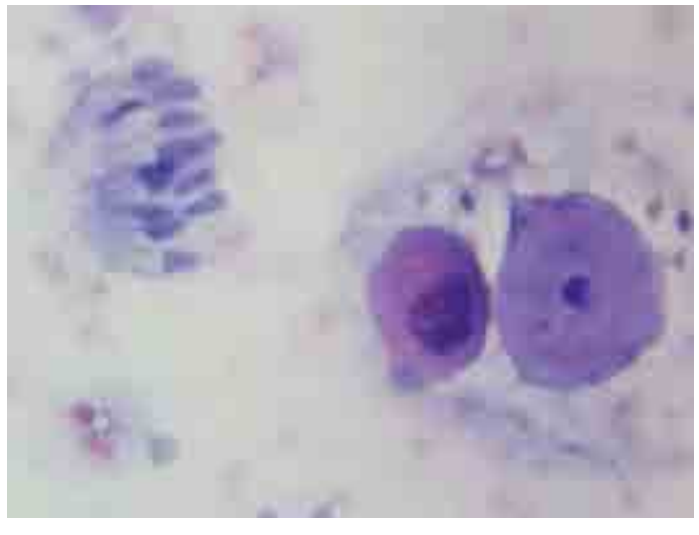

Figura 1. Célula con atipia correspondiente a lesión intraepitelial escamosa de alto grado y células endocervicales en íntima cercanía. Coloración de Papanicolaou, 400x. Fotografía tomada por una de las patólogas investigadoras, correspondiente a una de las láminas del estudio.

al concepto ampliamente aceptado del origen de las lesiones de alto grado en la zona de transformación que tienden a extenderse hacia el endocervix y a comprometer las glándulas endocervicales [28].

\section{Análisis estadístico}

Los datos se anotaron en un instrumento de recolección de información estructurado, identificando cada caso con un número, y fueron almacenados en una hoja de cálculo de Microsoft Excel Windows Vista ${ }^{\circledR}$ (Microsoft, Washington, Estados Unidos) y analizados utilizando el paquete estadístico SPSS $16.0^{\circledR}$ (IBM, Nueva York, Estados Unidos) en el cual se obtuvieron proporciones y se realizó el diseño 
de las tablas y las gráficas. Se utilizó el estadístico $X^{2}$, considerando significativo un valor de $p$ menor que 0,05 .

\section{Resultados}

\section{Características de las citologías cervicales estudiadas}

En el período comprendido entre 2009 y 2012 se estudiaron 32.287 citologías cervicales de las cuales 1.323 fueron anormales. Se eliminaron 36 extendidos atróficos, quedando incluidos 1.287 extendidos citológicos anormales, de los cuales el mayor porcentaje $(66,1 \%)$ correspondió a lesiones escamosas de bajo grado, seguidas por las atipias en células escamosas de significado indeterminado $(17,1 \%)$, las lesiones de alto grado $(13,2 \%)$ y, por último, las atipias en células escamosas que podrían indicar lesión de alto grado o mayor $(3,6 \%)$.

\section{Análisis de la presencia de componente de zona de transformación}

De los 1.287 extendidos anormales estudiados se encontró en mayor frecuencia la presencia de células endocervicales o metaplásicas $(74,7 \%)$ que la ausencia de estas $(25,3 \%)$ (véase tabla 1$)$. A medida que aumentó el grado de la lesión se

\begin{tabular}{lll}
\hline \multicolumn{3}{l}{ Tabla 1. Presencia de células endo- } \\
cervicales en extendidos anormales
\end{tabular}
incrementó el porcentaje de presencia de células endocervicales, con una diferencia estadísticamente significativa, observándose el componente de zona de transformación con mayor frecuencia en aquellos extendidos con lesiones más severas (véase tabla 2 ).

\section{Análisis de la cercanía}

\section{entre las células endocervicales y las anormales}

En general, en el 48,8\% de los 961 extendidos anormales con componente de la zona de transformación las células endocervicales o metaplásicas se ubicaron en un campo microscópico diferente al de las células anormales. Por su parte, las células endocervicales y anormales coincidieron en el mismo campo microscópico en el $28,7 \%$ de estas láminas cuando se examinaron con el mayor aumento (40X), en el $19,1 \%$ con el de mediano aumento $(10 \mathrm{X})$ y en el $3,3 \%$ con el panorámico (4X).

Cuando los resultados de las citologías se reclasificaron en lesión intraepitelial escamosa de bajo grado/atipias en células escamosas de significado indeterminado y lesión intraepitelial escamosa de alto grado/atipias en células escamosas que podrían indicar lesión de alto grado, en los 961 extendidos anormales con presencia de células endocervicales se encontró que la localización de las células 


\begin{tabular}{|c|c|c|c|c|c|}
\hline \multirow{2}{*}{ Resultado citológico } & \multirow{2}{*}{ N. ${ }^{\circ}$ de casos } & \multicolumn{4}{|c|}{ Presencia de células endocervicales/metaplásicas } \\
\hline & & No & $\%$ & Sí & $\%$ \\
\hline $\begin{array}{l}\text { Atipias en células escamosas } \\
\text { de significado indeterminado } \\
\text { (ASC-US) }\end{array}$ & 220 & 62 & 28,2 & 158 & 71,8 \\
\hline $\begin{array}{l}\text { Lesión intraepitelial escamosa } \\
\text { de bajo grado (LIEBG) }\end{array}$ & 851 & 235 & 27,6 & 616 & 72,4 \\
\hline $\begin{array}{l}\text { Atipias en células escamosas } \\
\text { que podrían indicar lesión de } \\
\text { alto grado (ASC-H) }\end{array}$ & 46 & 8 & 17,4 & 38 & 82,6 \\
\hline $\begin{array}{l}\text { Lesión intraepitelial escamosa } \\
\text { de alto grado (LIEAG) }\end{array}$ & 170 & 21 & 12,4 & 149 & 87,6 \\
\hline Total & 1.287 & 326 & 25,3 & 961 & 74,7 \\
\hline
\end{tabular}

endocervicales y las anormales en el mismo campo microscópico fue mayor en las lesiones de alto grado, independientemente del poder del objetivo utilizado. Esta diferencia se hizo mayor al disminuir el tamaño del campo microscópico, evidenciándose la estrecha relación entre las células anormales y las endocervicales (véase tabla 3 ).

\section{Discusión}

Un resultado importante de este estudio es el porcentaje de citologías con representación de la zona de transformación inferior al parámetro aceptado, que establece que más del $80 \%$ de los extendidos deben presentar este componente [18]. En estudios en los que se describe la presencia de células de la zona de transformación en extendidos anormales, como los de Elías y colaboradores (1983) [29], Vooijs y colaboradores (1985) [10], y Leung y colaboradores (2009) [23], se ha reportado su presencia entre el $77,8 \%$ y el $98,5 \%$ de los extendidos; cifras superiores a los hallazgos del presente estudio.

La proporción del componente de la zona de transformación en este estudio es directamente proporcional al grado de la lesión detectada, de manera similar a lo reportado por Leung y colaboradores (2009) [23], quienes encontraron una mayor frecuencia de presencia de este componente a medida que aumentaba el grado de la lesión, con presencia de células endocervicales en el $88 \%$ de los extendidos en los que se detectó lesión intraepitelial escamosa de alto grado y en el $74 \%$ de los que tenían lesión intraepitelial escamosa de bajo grado. Contrariamente, Bos 


\begin{tabular}{|c|c|c|c|c|c|c|}
\hline \multicolumn{7}{|l|}{ Bajo poder $(4 x)$} \\
\hline \multirow[t]{2}{*}{ Resultado citología } & \multirow[t]{2}{*}{ N. ${ }^{\circ}$ de casos } & \multicolumn{4}{|c|}{$\begin{array}{l}\text { Presencia de células de la zona de trans- } \\
\text { formación y células anormales en el mismo } \\
\text { campo }\end{array}$} & \multirow[t]{2}{*}{ Probabilidad $X^{2}$} \\
\hline & & No & $\%$ & Sí & $\%$ & \\
\hline No alto grado & 774 & 273 & 35,3 & 501 & 64,7 & \multirow{3}{*}{0,000} \\
\hline Alto grado & 187 & 29 & 15,5 & 158 & 84,5 & \\
\hline Total & 961 & 302 & 31,4 & 659 & 68,6 & \\
\hline \multicolumn{7}{|l|}{ Mediano poder $(10 x)$} \\
\hline \multirow[t]{2}{*}{ Resultado citología } & \multirow[t]{2}{*}{ N. ${ }^{\circ}$ de casos } & \multicolumn{4}{|c|}{$\begin{array}{l}\text { Presencia de células de la zona de trans- } \\
\text { formación y células anormales en el mismo } \\
\text { campo }\end{array}$} & \multirow[b]{2}{*}{ Probabilidad $X^{2}$} \\
\hline & & No & $\%$ & Sí & $\%$ & \\
\hline No alto grado & 774 & 312 & 40,3 & 462 & 59,7 & \multirow{3}{*}{0,000} \\
\hline Alto grado & 187 & 33 & 17,6 & 154 & 82,4 & \\
\hline Total & 961 & 345 & 35,9 & 616 & 64,1 & \\
\hline \multicolumn{7}{|l|}{ Alto poder (40x) } \\
\hline \multirow[t]{2}{*}{ Resultado citología } & \multirow[t]{2}{*}{ N. ${ }^{\circ}$ de casos } & \multicolumn{4}{|c|}{$\begin{array}{l}\text { Presencia de células de la zona de trans- } \\
\text { formación y células anormales en el mismo } \\
\text { campo }\end{array}$} & Probabilidad $X^{2}$ \\
\hline & & No & $\%$ & Sí & $\%$ & \\
\hline No alto grado & 774 & 519 & 67,1 & 255 & 32,9 & \multirow{3}{*}{0,000} \\
\hline Alto grado & 187 & 72 & 38,5 & 115 & 61,5 & \\
\hline Total & 961 & 591 & 61,5 & 370 & 38,5 & \\
\hline
\end{tabular}

y colaboradores (2001) [22] no reportaron diferencias entre la severidad de la lesión y la presencia o ausencia de células endocervicales.

El resultado más llamativo de este estudio se relaciona con la proximidad entre las células endocervicales o metaplásicas y las células anormales en los extendidos citológicos, donde se hizo evidente la íntima relación de este componente con las células anormales con atipias de alto grado, mientras que en las lesiones de bajo grado esta cercanía no fue tan estrecha. Esta relación, hasta nuestro conocimiento, no había sido documentada en estudios de extendidos de citología convencional.

Este hallazgo puede estar relacionado con la historia natural de la infección por el virus del papiloma humano y el desarrollo de las lesiones intraepiteliales del cérvix, debido a que los virus de bajo riesgo usan la maduración del epitelio para replicarse y, por esto, los cambios citopáticos (coilocitosis) que producen se observan en las células escamosas diferenciadas, con una expansión horizontal en el epitelio, a diferencia de los virus de alto riesgo que se integran al genoma de las células 
basales de la unión escamocolumnar y desde allí transforman todo el espesor del epitelio en forma vertical y con tendencia al compromiso endocervical $[30,31]$.

A la luz de estos hallazgos sería lógico pensar que los extendidos sin componente de la zona de transformación son menos sensibles para la detección de alteraciones epiteliales del cérvix; sin embargo, los resultados alrededor de este tema son controversiales. En el análisis realizado por Elumir-Tanner y Doraty (2011) [32] sobre este aspecto se reporta que su ausencia no aumenta el riesgo de presencia de alteraciones citológicas en tamizajes posteriores. De modo que, ante resultados de citologías sin presencia de este componente, su manejo debe ser contextualizado a las condiciones locales.

Por ejemplo, en los países desarrollados con programas de alta calidad, en los que suele estar garantizada una óptima toma de muestra, la ausencia de representación de la zona de transformación se atribuye a las condiciones clínicas del cérvix relacionadas con su localización anatómica, el período de embarazo o la realización de procedimientos quirúrgicos previos que son de conocimiento del clínico quien, en últimas, determina la satisfactoriedad de la muestra al relacionarla con los antecedentes de la paciente [17].

En nuestro medio la ausencia del componente de la zona de transformación no es detectada ni asociada a los antecedentes de la paciente, debido a que el clínico, en la mayoría de los casos, no accede a los resultados negativos y no tiene la oportunidad de considerar la necesidad de repetir el estudio. Este esquema de manejo dentro del Programa de Control del Cáncer de Cuello Uterino puede llevar a no detectar los falsos negativos, lo que se evidencia en el alto porcentaje de casos de cáncer invasor encontrado en pacientes con antecedente reciente de citología negativa $[19,33]$.

\section{Conclusiones}

Existe una mayor frecuencia de extendidos citológicos anormales con presencia de células de la zona de trasformación del cérvix, que sin ella. Además, hay una asociación entre la presencia de este componente y el grado de la lesión, siendo más frecuente en los extendidos con lesiones de alto grado, donde además las células endocervicales o metaplásicas se encuentran íntimamente relacionadas con las células anormales.

\section{Bibliografía}

1. Munoz N, Bravo LE. Epidemiology of cervical cancer in Colombia. Colomb Med (Cali) 2012; 43: 298-304.

2. Murillo R. [Cervical cancer control in Colombia: achievements and challenges of cytology based programs]. Biomedica 2008; 28: 467-470.

3. Richart RM. A theory of cervical carcinogenesis. Obstet Gynecol Surv 1969; 24: 874-879.

4. Hans N, Cave AJ, Szafran O, Johnson G, Glass 
A, Spooner GR, et al. Papanicolaou smears: to swab or not to swab. Can Fam Physician 2007; 53: $1328-1329$.

5. Lacruz C. Nomenclatura de las lesiones cervicales. (De Papanicolau a Bethesda 2001). Rev Esp Esp 2003; 36: 5-10.

6. Papanicolaou GN. Cytologic studies in diagnosis of carcinoma. J Int Coll Surg 1954; 21: 419-426.

7. Arbyn $\mathbf{M}$, Bergeron $\mathbf{C}$, Klinkhamer $\mathbf{P}$, MartinHirsch P, Siebers AG, Bulten J. Liquid compared with conventional cervical cytology: a systematic review and meta-analysis. Obstet Gynecol 2008; 111: $167-177$

8. Karimi-Zarchi M, Peighmbari F, Karimi $\mathbf{N}$ Rohi M, Chiti Z. A Comparison of 3 Ways of Conventional Pap Smear, Liquid-Based Cytology and Colposcopy vs Cervical Biopsy for Early Diagnosis of Premalignant Lesions or Cervical Cancer in Women with Abnormal Conventional Pap Test. Int J Biomed Sci 2013; 9: 205-210.

9. Lazcano-Ponce EC, de Ruiz PA, Lopez-Carrillo L, Vazquez-Manriquez ME, Hernandez-Avila M. Quality control study on negative gynecological cytology in Mexico. Diagn Cytopathol 1994; 10: 10-14.

10. Vooijs PG, Elias A, van der Graaf $\mathbf{Y}$, Veling S. Relationship between the diagnosis of epithelial abnormalities and the composition of cervical smears. Acta Cytol 1985; 29: 323-328.

11. Sultana F, English DR, Simpson JA, Canfell K, Gertig DM, Saville M. High-grade cervica abnormalities and cervical cancer in women following a negative Pap smear with and without an endocervical component: a cohort study with 10 years of follow-up. Int J Cancer 2014; 135 1213-1219.

12. Marchand L, Mundt M, Klein G, Agarwal SC. Optimal collection technique and devices for quality pap smear. WMJ 2005; 104: 51-55.

13. Valente PT, Schantz HD, Trabal JF. The determination of Papanicolaou smear adequacy using a semiquantitative method to evaluate cellularity. Diagn Cytopathol 1991; 7: 576-580.

14. Fokke HE, Salvatore CM, Schipper ME, Bleker OP. The quality of the Pap smear. Eur J Gynaecol Oncol 1992; 13: 445-448.

15. The 1988 Bethesda System for reporting cervical/ vaginal cytological diagnoses. National Cancer Institute Workshop. JAMA 1989; 262: 931-934.

16. The Bethesda System for reporting cervical/ vaginal cytologic diagnoses: revised after the second National Cancer Institute Workshop, April 29-30, 1991. Acta Cytol 1993; 37: 115-124.

17. Solomon D, Davey D, Kurman R, Moriarty A, O'Connor D, Prey M, et al. The 2001 Bethesda System: terminology for reporting results of cervical cytology. JAMA 2002; 287: 2114-2119.

18. Pritchard J. Quality Assurance Guidelines for the Cervical Screening Programme - Report of a Working Party Convened by the NHS Cervical Screening Programme: NHSCSP; 1996.

19. Pineros M, Cendales R, Murillo R, Wiesner $\mathbf{C}$ Tovar S. [Pap test coverage and related factors in Colombia, 2005]. Rev Salud Publica (Bogota) 2007; 9: 327-341.

20. Narine N, Young $\mathbf{W}$. Transformation zone sampling rate is a useful performance indicator for practitioners collecting cervical samples using SurePath liquid-based cytology system. Cytopathology 2007; 18: 220-224.

21. Young $\mathbf{w}$. Comparison of transformation zone sampling rates--a potentially useful indicator of smear taker performance. Cytopathology 2000; 11: 116-123.

22. Bos $A B$, van Ballegooijen $M$, Elske van den Akker-van Marle $M$, Hanselaar AG, van Oortmarssen GJ, Habbema JD. Endocervical status is not predictive of the incidence of cervical cancer in the years after negative smears. Am J Clin Pathol 2001; 115: 851-855.

23. Leung KM, Lam M, Lee JW, Yeoh GP, Chan KW. The significance of endocervical cells and metaplastic squamous cells in liquid-based cervical cytology. Diagn Cytopathol 2009; 37: 241-243.

24. Murillo R. La prevención del cáncer: más allá de las pruebas de tamizaje. Rev Colomb Cancerol 2003; 7: 23-33.

25. Selvaggi SM, Guidos BJ. Endocervical component: is it a determinant of specimen adequacy? Diagn Cytopathol 2002; 26: 53-55.

26. Celasun B. Presence of endocervical cells and number of slides in cervicovaginal smears: differences in performance between gynecologists. Acta Cytol 2001; 45: 730-734.

27. Arbyn $M$, Herbert A, Schenck $U$, Nieminen P, Jordan J, McGoogan E, et al. European guidelines for quality assurance in cervical cancer screening: recommendations for collecting samples for conventional and liquid-based cytology. Cytopathology 2007; 18: 133-139.

28. Kurman RJ. Tumors of the Cervix, Vagina and Vulva: AFIP Atlas of Tumor Pathology Series. Vol. 13; 2011.

29. Elias A, Linthorst G, Bekker B, Vooijs PG. The significance of endocervical cells in the diagnosis of cervical epithelial changes. Acta Cytol 1983; 27: 225-229.

30. Doorbar J, Quint W, Banks L, Bravo IG, Stoler M, Broker TR, et al. The biology and life-cycle of human papillomaviruses. Vaccine 2012; 30 Suppl 5: F55-70

31. Baak JP, Kruse AJ, Robboy SJ, Janssen EA van Diermen B, Skaland I. Dynamic behavioura interpretation of cervical intraepithelial neoplasia with molecular biomarkers. J Clin Pathol 2006; 59: $1017-1028$

32. Elumir-Tanner L, Doraty M. Management of Papanicolaou test results that lack endocervical cells. CMAJ 2011; 183: 563-568.

33. Murillo R, Cendales R, Wiesner C, Pineros $\mathbf{M}$, Tovar S. [Effectiveness of cytology-based cervical cancer screening in the Colombian health system]. Biomedica 2009; 29: 354-361. 